\title{
DETERMINATION OF TECHNICAL AND ECONOMIC EFFICIENCY OF EQUIPMENT FOR MASS BREEDING OF ENTOMOPHAGES
}

\author{
Valentyna Krutyakova $^{1}$, Igor Bespalov ${ }^{1}$, Adolfs Rucins ${ }^{2}$, Volodymyr Bulgakov ${ }^{3}$ \\ ${ }^{1}$ Engineering and Technology Institute "Biotechnica", Ukraine; \\ ${ }^{2}$ Latvia University of Life Sciences and Technologies, Latvia; \\ ${ }^{3}$ National University of Life and Environmental Sciences of Ukraine, Ukraine \\ arucins@ltk.lv
}

\begin{abstract}
The existing practice of creating the equipment for industrial production of entomophages is usually based on the development of the working design documentation for already tested mock-ups and experimental samples of the laboratory equipment obtained as a result of the research work on introduction of insects into culture and their breeding. However, such an approach is rather expensive. This study is aimed at a methodological provision of the stage of the engineering design of technological complexes for industrial production of entomophages, the main characteristics of which are determined by the design of the cages for insects. The proposed scientifically based scaling methodology substantiates a possibility and conditions to ensure pre-set values of the abiotic and the biotic factors of the cage models when their areas are changed. Criteria are justified for a complex assessment of various technical solutions by way of minimising the relative technological prime cost of manufacturing the cages and boxes in which they are implemented. Analytical dependences were obtained and investigated of the technological cost of a set of cages and boxes for the cultivation of lacewings on their areas. It has been established that increasing the area of the cage for lacewings from 0.0625 to $0.125 \mathrm{~m}^{2}$ reduces its cost from 1.43 to $1.06 \mathrm{UAH}$-(insect) ${ }^{-1}$, and increasing the area of the box for cages from 0.3 to $0.6 \mathrm{~m} 2$ reduces its cost from 0.55 to $0.04 \mathrm{UAH}$. (insect) ${ }^{-1}$. In order to minimise the prime cost, it is necessary to increase the horizontal cross-sectional areas of cages and boxes to values limited by the ease of use or the structural strength. The developed methodologies were tested during the design of a technological complex for the production of the gold-eye entomophagy, including the prototype cages. In comparison with the existing equipment, a 50-60\% improvement in the economic indicators has been achieved.
\end{abstract}

Keywords: production, entomophages, equipment, simulation.

\section{Introduction}

The production and use of entomophages for the biological protection of plants from pests is one of the main mechanisms for the ecologisation of agriculture [1]. This production is based on appropriate technological equipment, which is designed for several dozen insects and ticks [2], including for the well-known entomophage of lacewing [3], an example of which is being studied according to the developed new technique.

The existing practice of creating equipment for the industrial production of entomophages is usually based on the development of working design documentation for already tested mock-ups and experimental samples of laboratory equipment, which were obtained as a result of research work on introducing insects into the culture and breeding.

However, when the production volumes increase to an industrial level, this approach becomes economically inefficient. Analysis of the existing equipment [5-7] shows that each stage of the development of an insect occurs in a certain cage, in the closed space of which the necessary living conditions for insects are created. Obviously, the design of the cages created during the development of the breeding technologies in the research laboratory will not be optimal at the factory for the production of entomophages.

There a need arises for a system engineering (engineering) designing [8], which allows implementation of specified biotechnological requirements for breeding insects in various variants of the equipment with the choice of the most efficient of them. One of the methods for solving this problem is mathematical simulation of the technical and economic efficiency of the main equipment for breeding entomophages.

The purpose of simulation is to determine the dependence of economic characteristics on the structural technical parameters of the technological equipment. This will allow to find the values of the design parameters that provide maximum economic efficiency of the equipment. 


\section{Materials and methods}

When designing the production on the basis of the existing laboratory technology for the mass breeding of specific insects, the technological process is predetermined and unchanged. But its technological (regulatory) indicators can be implemented in various design solutions of the equipment. As a result of our research, we developed a technique for scaling cages, which allows to change their sizes in compliance with the basic biotechnological requirements. It was substantiated that the determining condition for scaling is the invariance of the height of the insect layer with the food substrate, which sets the height of the cage, as well as the surface or bulk density of insects. Other abiotic parameters that must be preserved when scaling include temperature and the air humidity. This condition is met by ensuring the constancy of the ratio of the area of the ventilation openings to the area of the cage in the base and scalable versions [9].

The analysis of the industrial equipment for the production of common entomophages $[9 ; 10]$ shows that each stage (or several stages) of insect ontogenesis proceeds in a cage, which is usually parallelepiped of the height $h_{c}$ and area $S_{c}$. Cages in the amount of $n_{c}$ are placed in the technological box, the number of boxes is $n_{a .}$. The totality of the indicated quantities of cages and boxes makes up a set of equipment at this stage of ontogenesis. The capacity of the set per a breeding cycle is determined by the expression:

$$
Q=n_{c} \cdot n_{a} \cdot Q_{c}=d \cdot S,
$$

where $Q$ - assigned efficiency of the set for the insects;

$S$ - total area for keeping insects, $\mathrm{m}^{2}$;

$d$-surface density of insects in the cage, $\mathrm{pcs}^{-2}$.

The geometric characteristics of the cage and box can be determined from such expressions:

$$
V_{o}=S_{c} \cdot h_{c}, \quad V_{a}=S_{a} \cdot h_{a}=\frac{n_{o} \cdot S_{c} \cdot h_{c}}{\eta},
$$

where $V_{o}, V_{a}-$ volume of the cage and box, respectively;

$\eta=h_{v} \cdot V_{o} \cdot\left(V_{a}\right)^{-1}-$ coefficient of filling the box with cages;

$h_{a}$ - height of the box;

$S_{a}$ - cross-sectional area of the box (the shelf area).

From expressions (1) and (2) is determined:

$$
n_{a}=\frac{S \cdot h_{c}}{\eta \cdot S_{a} \cdot h_{a}} .
$$

When updating the equipment, determination of the economic efficiency is most often carried out through the technological cost of manufacturing the equipment $K$, which consists of the cost of the materials $-M$, salary $-T$, the energy carriers $-E$.

Therefore, a need arises to develop such a new mathematical model that could be successfully used not to calculate specific economic indicators, but to determine the possibility and direction of optimisation of the equipment for systems engineering in the field of biotechnology, in particular, for efficient cultivation of entomophages.

The energy costs at the designing stage can be determined only through the coefficients of communication with other cost items. From the experience we have accepted:

$$
E=k_{e} \cdot T,
$$

from where:

$$
K=M+k \cdot T
$$

where $K$ - technological cost of manufacturing a unit of equipment;

$k=1+k_{e}-$ cost conversion factor, which we will consider constant in each variant of the equipment. 
The technological cost of manufacturing a set of the equipment, which consists of cages and boxes:

$$
\begin{gathered}
K=K_{c}+K_{a}, \\
K_{c}=n_{c} \cdot n_{a}\left(M_{c}+k_{c} \cdot T_{c}\right), \\
K_{a}=n_{a}\left(M_{a}+k_{a} \cdot T_{a}\right),
\end{gathered}
$$

where $n_{c}, n_{a}$ - number of cages and boxes, respectively.

For the analysis costs are usually used which are given in the conventional unit of production:

$$
K^{\prime}=\frac{K}{Q}=\frac{K}{d \cdot S},
$$

where $K^{\prime}$ - reduced cost of manufacturing the equipment.

From expressions (3) - (8) we obtain:

$$
\begin{gathered}
K_{c}^{\prime}=\frac{M_{c}+k_{c} \cdot T_{c}}{d \cdot S_{c}}, \\
K_{a}^{\prime}=\frac{\left(M_{a}+k_{a} \cdot T_{a}\right) \cdot h_{c}}{d \cdot \eta \cdot S_{a} \cdot h_{a}} .
\end{gathered}
$$

Further we will use only the reduced cost, let us omit the bar in designation $K^{\prime}$.

When simulating, an assumption was made that the monetary costs of manufacturing the apparatus or its parts are described by linear functions of the determining geometric parameter:

$$
\begin{gathered}
M=m \cdot S, \\
T=v \cdot S, \\
T=u \cdot L,
\end{gathered}
$$

where $M, T-$ costs for manufacturing of the part, $\mathrm{UAH}$;

$m, v$ - conversion factors, $\mathrm{UAH} \cdot \mathrm{m}^{-2}$;

$u$ - conversion factor, $\mathrm{UAH} \cdot \mathrm{m}^{-2}$

$S$ - specific surface area, $\mathrm{m}^{2}$;

$L-$ conditional linear size of the part, $\mathrm{m}$.

This assumption has sufficient accuracy when comparing variants of the same part or apparatus, which have the same shape and materials, but differ in size.

\section{Results and discussion}

The parameter to be optimized (minimised) was the reduced technological cost of manufacturing a set of cages and boxes $(10,11)$. We define the main dependencies between the economic and design parameters of the equipment that were the basis of the models. From the given set of parameters in $(10,11)$ we choose as independent $S_{c}, S_{a}$ can be changed for optimisation.

During the design, the following biological and technical requirements were specified [9-10]: $h_{c}$ - the cage height, $\mathrm{m} ; h_{a}$ - the height of the box, $d$ - the surface density of insects, individuals $\mathrm{m}^{-2}$; from the technical task $Q$ - the productivity of the set, insects per cycle.

The model of the cost for making the cage. The cage is a closed volume, which contains insects at different stages of ontogenesis. It is made of plastic, consists of a shell, a bottom, a removable cover. The body is parallelepiped of the height $h_{c}$. Its bottom area is $S_{c}$, which is further considered to be square with the side length, to simplify the formulas $l_{c}=\sqrt{S_{c}}$.

The costs of the cage materials according to (12) are calculated in proportion to the area of its side surface and are averaged in the case of various materials: 


$$
M_{c}=m_{c}\left(2 S_{c}+4 h_{c} \sqrt{S_{c}}\right) \text {. }
$$

The labour costs (14) are calculated in proportion to the doubled sum of the lengths of all the edges of the body blanks on which the blanks are cut, glued (welded) or bent and mechanically fixed:

$$
T_{c}=2 \cdot u_{c} \cdot\left(8 \sqrt{S_{c}}+4 h_{c}\right)
$$

We substitute the obtained in (10):

$$
K_{c}=\frac{2 m_{c}}{d}+\frac{1}{d \cdot \sqrt{S_{c}}}\left(4 h_{c} \cdot m_{c}+16 u_{c} \cdot k_{c}\right)+\frac{8 h_{c} \cdot u_{c} \cdot k_{c}}{d \cdot S_{c}}
$$

The model of the production cost of the box. The box has a parallelepiped shape with a conditionally square base and consists of two parts: a shell and a shelving structure made of shelves for placing cages.

The costs of the shell materials $M_{S}$, according to (12), are calculated in proportion to the area of its lateral surface and averaged in the case of various materials:

$$
M_{S}=m_{S}\left(2 S_{a}+4 h_{a} \cdot \sqrt{S_{a}}\right) .
$$

The labour costs (14) are calculated in proportion to twice the sum of the lengths of all the edges of the shell blanks:

$$
T_{S}=2 u_{S}\left(8 \cdot \sqrt{S_{a}}+4 h_{a}\right)
$$

The costs of the material of the rack structure (12) from the shelves are calculated in proportion to the area of the shelves:

$$
M_{h}=m_{h} \cdot n_{h} \cdot S_{a},
$$

where $n_{h}$ - number of shelves in the box (12).

The labour costs for the rack structure (13) are calculated in proportion to the area of the shelves:

$$
T_{h}=v_{h} \cdot n_{h} \cdot S_{a} \text {. }
$$

We substitute everything in (11) for the box:

$$
K_{a}=\left[M_{S}+M_{h}+k_{a}\left(T_{S}+T_{h}\right)\right] \cdot \frac{h_{c}}{d \cdot \eta \cdot S_{a} \cdot h_{a}}=\frac{h_{c} \cdot \bar{K}_{a}}{d \cdot \eta \cdot h_{a}},
$$

where

$$
\begin{aligned}
& \bar{K}_{a}=\left(2 m_{S}+m_{h} \cdot n_{h}+k_{a} \cdot v_{h} \cdot n_{h}\right)+ \\
& +\frac{1}{\sqrt{S_{a}}}\left(4 m_{S} \cdot h_{a}+16 k_{a} \cdot u_{S}\right)+\frac{1}{S_{a}}\left(8 k_{a} \cdot u_{S} \cdot h_{a}\right) .
\end{aligned}
$$

The developed model of the reduced technological cost for manufacturing cages and boxes was used in the system design of a new technological complex for the production of ordinary lacewing. Earlier, we carried out the scientific and research work on the development of a technology and the equipment [8] for lacewing, which was chosen as the basic option when comparing the technical and economic efficiency.

As an example, let us consider simulation of a cage for the lacewing imago. Fig. 1 shows the mock-up options for cages with different $S_{C}$ area, which were made to study the impact of $S_{C}$ on the economic efficiency. Cages 4 are made of acrylic and are shown without covers. The lid 5 of the cage is shown in the background, from the inside the lid is glued with a black cloth on which the imago of the lacewing lay their eggs. Cages 1 and 2 were used for laboratory dilution of the lacewing. 


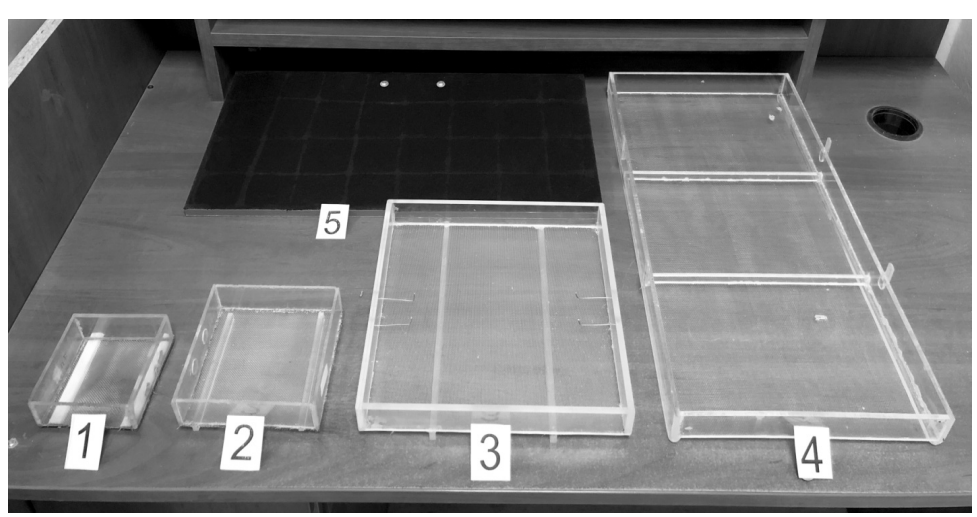

Fig. 1. Variants of the cages for the imago lacewing for the research of the economic efficiency of their manufacture

Cage 3 with the dimensions $25-25 \mathrm{~cm}$ was used in the basic industrial set. For it by the wellknown direct calculation method the costs $K, M, T, E$ were calculated which allowed using formulas $(4,15-21)$ to calculate the average values of the coefficients $k, m, u, v$. In a similar manner the conversion factors were calculated for the box of a standard design. The following are the values of the model parameter:

- of the cage: $h_{c}=0.03 \mathrm{~m}, d=11200$ insects $\mathrm{m}^{-2}, m_{c}=1199 \mathrm{UAH} \cdot \mathrm{m}^{-2}, u_{c}=186.3 \mathrm{UAH} \cdot \mathrm{m}^{-1}$, $k_{c}=1.03$;

- of the box: $h_{a}=1.95 \mathrm{~m}, \quad n_{h}=5, \eta=0.26, \quad m_{S}=65.9 \mathrm{UAH} \cdot \mathrm{m}^{-2}, \quad m_{h}=220 \mathrm{UAH} \cdot \mathrm{m}^{-2}$, $u_{S}=72.6 \mathrm{UAH} \cdot \mathrm{m}^{-1}, v_{h}=440 \mathrm{UAH} \cdot \mathrm{m}^{-2}$.

Figures 2 and 3 show the dependences of the technological cost of the cages and boxes on their areas, which are calculated on a PC using formulas $(17,22,23)$.

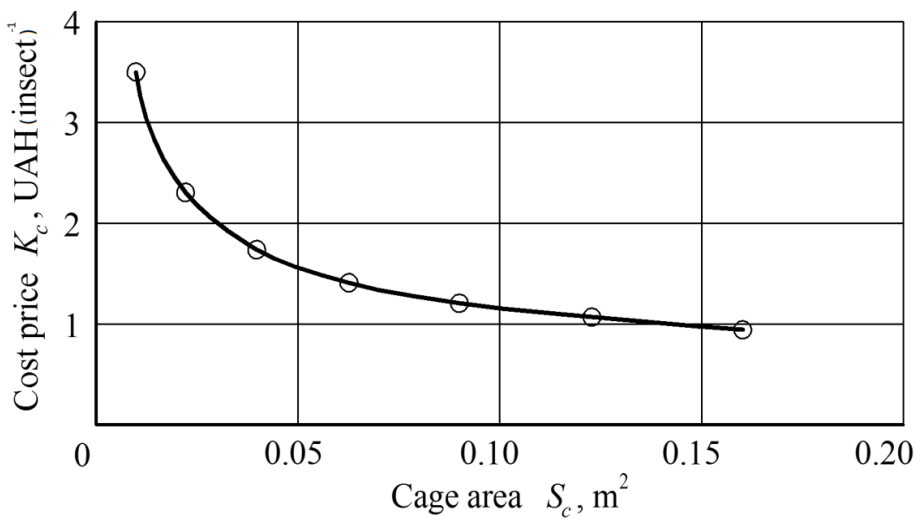

Fig. 2. Dependence of the technological cost of a set of cages of the imago lacewings on the area of the cage

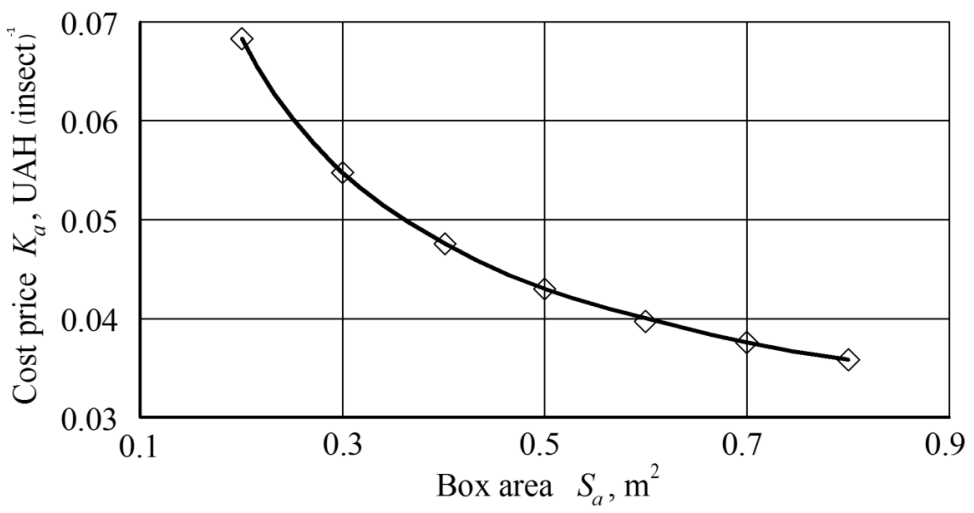

Fig. 3. Dependence of the technological cost of a set of boxes for cages of the imago lacewings on the area of the box 
Analysis of the mathematical formulas and graphs shows that the reduced technological cost of manufacturing a set of cages and boxes monotonously decreases with an increase in their area. This proves the economic expediency of increasing the area of the cage and the box to their maximum possible dimensions, which are determined by the ergonomic requirements for comfortable work of the staff. Therefore, an imago cage (Fig. 1, Cages 4) was chosen with dimensions of $25 \times 50 \mathrm{~cm}$ and an area of $0.125 \mathrm{~m}^{2}$, with a prime cost of $K_{c}=1.06 \mathrm{UAH} \cdot\left(\right.$ insect) ${ }^{-1}$ (see Fig. 2). Compared with the base cage of the old complex $25 \times 25 \mathrm{~cm}$, area $0.0625 \mathrm{~m}^{2}$, the prime cost $1.43 \mathrm{UAH}$ (insect) ${ }^{-1}$, a reduction of the reduced technological cost by $25.9 \%$ was achieved. Comparison of the numerical values of $K_{c}$, $K_{a}$ in Figs. 2, 3 shows that $K_{a}$ is approximately $4 \%$ of $K_{c}$, i.e. the impact of the component $K_{a}$ upon the total cost of the set $K(5)$ is sufficiently small. This regularity is explained by the fact that the number of cages significantly exceeds the number of boxes, and indicates that it is the design of the cage that determines the technical and economic efficiency of the entire set.

\section{Conclusions}

1. Approbation of the model during the system-and-technical designing of the technological complex for the production of an entomophage - the ordinary lacewing allowed to reduce by $26 \%$ the technological cost of manufacturing a set of cages and boxes.

2. By means of this model there is first substantiated the economic purposefulness of maximal increase of the area of individual cages and boxes for a given area of their set.

3. As a result of the research, it was found that increasing the area of the cage for the cultivation of lacewings from 0.0625 to $0.125 \mathrm{~m}^{2}$ reduces its cost from 1.43 to $1.06 \mathrm{UAH} \cdot$ (insect) $^{-1}$, and increasing the area of the box for cages from 0.3 to $0.6 \mathrm{~m}^{2}$.

\section{References}

[1] Van Lenteren J.C., Bolckmans K., Köhl J. Biological control using invertebrates and microorganisms: plenty of new opportunities. BioControl, 63, 2018, pp. 39-59.

[2] Cohen A.C. Ecology of Insect Rearing Systems: A Mini-Review of Insect Rearing Papers from 1906-2017. Advances in Entomology, 2018, Vol. 6 (2). pp. 86-115.

[3] Leppla N.C., Morales-Ramos J. A., Shapiro-Ilan D.I., Rojas M. G. Mass Production of Beneficial Organisms. Invertebrates and Entomopathogens. Elserier Inc., 2014, 16 p.

[4] Krutyakova V. Effective Technological Equipment for Mass Production of Entomophagous Insects and Mites Used for Biological Control. Journal of Agricultural Science and Technology, No 7, 2017, pp. 179-186.

[5] Morrison R.K., Ridgeway R.L. Improvements in Techniques and Equipment for Production of a Common Green Lacewing, Chrysopa Carnea. U.S. Department of Agriculture, Agricultural Research Service, 1976. 143 p.

[6] Tayyab M.M., Ehsan-ul-Haq, Naeemullah M., Shakeel M., Ashfaque M., Asmat un Nisa, Khan J. Economic rearing of chrysoperla carnea (stephens) (neuroptera: chrysopidae) in insectary. Pakistan J. Agric. Res., Vol. 26, No. 4, 2013, pp. 309-315.

[7] Хорошев А.Н. Основы системного проектирования технических объектов (Fundamentals of system design of technical objects). 2011, Москва, 119 с. (In Russian).

[8] Chris J. Design Methods, John Wiley and Sons Ltd., 1982, 336 p.

[9] Krutiakova V., Bespalov I., Bulgakov V., Viesturs D. Engineering design of industrial production of golden-eyed lacewings (L. chrysoperla carnea steph) in the programs of biological protection of plants. Engineering for rural development, Vol.18, 2019, pp.278-283.

[10]Быков В.В. Технология машиностроения. Проектирование (Engineering Technology. Design). Москва: МГУЛ, 2006, 320 с. (In Russian). 